\title{
A Hierarchical Search Space Refinement and filling for Exemplar based Image Inpainting
}

\author{
S.Padmavathi \\ Department of Information Technology, \\ Amrita School of Engineering, \\ Amrita Vishwa Vidyapeetham Coimbatore, India
}

\author{
K.P.Soman \\ Phd, Center of Computational Engineering \& \\ Networking, \\ Amrita School of Engineering, \\ Amrita Vishwa Vidyapeetham Coimbatore, India
}

\begin{abstract}
There are many real world scenarios where a portion of the image is damaged or lost. Restoring such an image without prior knowledge or a reference image is a difficult task. Image inpainting is a method that focuses on reconstructing the damaged or missing portion of images based on the information available from undamaged areas of the same image. The existing methods fill the missing area from the boundary. Their performance varies while reconstructing structures and textures and many of them restrict the size of the area to be inpainted. In this paper exemplar based inpainting is adopted in a hierarchical framework. A hierarchical search space refinement and hierarchical filling are proposed in this paper which increases the accuracy and handles the extra cost due to multi resolution processing in a better way. The former tries to select an exemplar suitable at all resolution levels restricting the search space from the lower resolution level. The later fills the region at lower resolution level whose results are taken to the higher levels. This makes the non boundary pixels known in the higher resolution level which in turn helps in search space refinement while increasing accuracy.
\end{abstract}

Keywords: Image inpainting, Hierarchical inpainting, exemplar based inpainting.

\section{INTRODUCTION}

A Photographic picture is a two dimensional image which can contain many objects. One may be interested in the object or scene that is hidden by another. For example, a beautiful picture may contain some letters written on it or a view of the Taj mahal maybe occluded or a historic painting may be torn or damaged. Here the picture below the letters, the occluded portion of the Taj mahal and the damaged portion of the painting needs to be restored. These become the missing portion or damaged portion of the image. The missing area is specified by the user based on color or a template called as mask. Image inpainting is an image restoration technique, which aims in automatically restoring missing information of image according to the information around damaged region. Inpainting is mainly used for heritage conservation, restoration of old photographs, removal of occlusions, special effects in photos and so on.

The inpainting problem can be considered as assigning the gray levels to the missing area called as $\Omega$ with the help of gray levels in the known area $\Phi$ of the same image as shown in Fig. 1, through the boundary $\partial \Omega$. The restoration of the structural information like edges or textural information like repeating patterns pose a major challenge for the inpainting techniques.

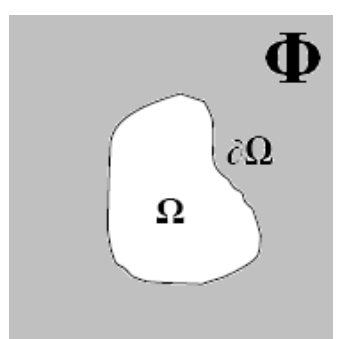

Fig. 1: Digital Image Inpainting

The technique presented in this paper is motivated by the Human visual system, in which a portion of the image blends with its neighborhood when viewed from various distances. Hence a similarity of the missing portion with the source region at various resolution levels is expected to provide a better result. The inpainting technique hence uses a patch based technique where the exemplars are extracted by sampling at various resolutions of an image. The set of closer matching patches from $\Phi$ are taken at lower resolution. This is then used as the starting set for a similar matching process at a higher resolution. Regions around each of the matches are then examined again at the higher resolution to find the best match with the boundary patch. The process is repeated until the final target image resolution; where upon the best match is used to generate the reconstructed pixel. This limits the search space, yet allows for some variation in best match from one scale to another. In another variation the missing area of the image at lower resolution is filled first using the exemplars obtained at that resolution. As a result, some intermediate pixels in the next higher resolution will be known. The process is repeated for different levels until all the pixels in the missing area are filled. By this process the coarser details which are represented in the lower resolution are filled first and then the finer details are filled. This results in better reconstruction.

\section{STATE OF ART}

Digital image inpainting is an ill posed problem and has many possible solutions. The inpainting problem has been discussed in [9] and [22]. Interpolation based solutions are discussed in [3] and [6]. Diffusion [1], level set, and other Partial Differential Equation models[21] have been Isuggested for solving the problem. The methods in [9], [10], [11], [12], [13], [17] and [19] uses Partial differential equations(PDE) for inpainting. These methods work well for inpainting smaller, non-textured regions. They perform inpainting by repeatedly convolving $\Omega$ using PDEs. These methods iteratively propagates information from $\delta \Omega$ into $\Omega$. The process is stopped when a steady state is reached. This procedure introduces noticeable blurring, when the pixel has significant contrast changes. The methods like standard concentric layer 
filling [19] and desired filling behavior [16] results in over shooting artifacts while propagating the linear structures. Inpainting can also be considered as an image synthesizing problem and hence texture synthesis methods could be adopted. Texture synthesis involves synthesizing an image that matches the appearance of a given texture[7]. The synthesized image may be of arbitrary size which should appear similar to the original image. Various texture synthesis method could be found in [2], [5], [8], [14], [15], and [18]. The method presented in [15] uses an image containing white random noise and synthesizes based on the original texture. A raster scan ordering is performed for all output pixels in the noisy image. The spatial neighborhood of each output pixel is compared to all possible neighborhoods in the original texture. The synthesized image appearance depends on the size and the shape of the neighborhood that is used. A combination of texture synthesis and digital inpainting has been tried in[4], [16] and [20]. They perform well in reconstructing textures but lag in structure propagatiom. Exemplar based techniques [23] and [24] could be considered as patch based texture synthesis method.. The algorithm in[24] assigns priority values of the fill-front based on the structural information surrounding it and fills $\Omega$ using exemplar patches in the order of priority. The purpose is to simultaneously preserve both texture and structure. In Exemplar based inpainting, it is difficult to produce reasonable results for synthesizing textures that do not have similar patches in the fill-front say $\delta \Omega$, and it does not handle depth ambiguities.

\section{HIERARCHICAL EXEMPLAR BASED IMAGE INPAINTING}

The exemplar based methods provide better result of larger area of inpainting and it does not blur the edges. However the textures on the boundary are propagated in to the inpainting area which collapses the larger structural information. This could be avoided by considering images at different resolution levels. Hierarchical methods at various image resolutions are capable of handling such a scenario. In this paper, two variations of such a Hierarchical method are proposed which uses the exemplar method as a basis. In both cases the input image is sub sampled to form images of lower spatial resolution. The algorithm starts with the lowest resolution and proceeds to higher resolution until the original image size is reached. These images form a hierarchy with actual sized image being considered to be at levell. The level number increases for each down sampling with the lowest spatial resolution image represented with the highest level number. The area to be inpainted is specified by the user through color selection or region selection in the actual sized image. This is called as mask. The pixels in the mask are then tagged and traced across the hierarchy during the inpainting process.

\subsection{Hierarchical Search Space Refinement}

The mask pixels in the lowest spatial resolution are traced and the boundary pixels are identified. The exemplar patches are formed with a boundary pixel as the center and including certain pixels from known area also called as source region $(\Phi)$ and certain pixels from mask also called as target region $(\Omega)$ as shown in Fig. 2. The known pixels in the patch form a basis for searching similar pixels in the known area.

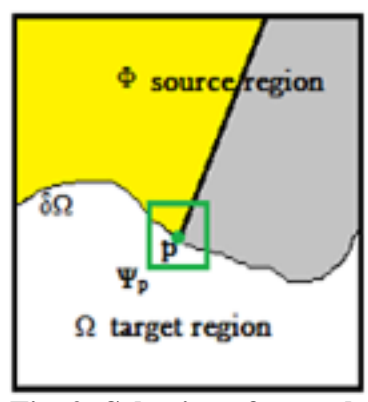

Fig. 2: Selection of a patch

In the traditional exemplar method an extensive search is done on the known area looking for closer matching patch for each exemplar patch. If the patch size is $n \times \mathrm{x} n$ and image size is $M$ $\mathrm{x} N$, then the search involves all possible $n \times n$ patches in the image. Neglecting the patches involving the mask area, the number of patches for each boundary pixel approximates to Eqn (1)

$$
(M-n)(N-n)-\left|\Omega_{i}\right|
$$

For an image at level 'i' with $\left|\Omega_{\mathrm{i}}\right|$ mask pixels, the number of patches will approximate to Eqn. (2). It is evident that as the image size decreases the possible patches to search also decreases.

$$
\left(\frac{M}{2^{i}}-n\right)\left(\frac{N}{2^{i}}-n\right)-\left|\Omega_{i}\right|
$$

However if the process is repeated for each level then the number of patches will be more as given by the eqn(3).

$$
\sum_{i=1}^{L}\left(\frac{M}{2^{i}}-n\right)\left(\frac{N}{2^{i}}-n\right)-\left|\Omega_{i}\right|
$$

In order to reduce the search cost, the closest matching patches up to a certain threshold are identified and their central locations are stored for each boundary pixel. In the next higher level, the boundary is again identified and the process is repeated for newly popped boundary pixels. The search involving the previous level boundary pixels as patch center is restricted to a small neighborhood of the locations stored earlier. The list is then updated and the process continues until the actual sized image where instead of storing the locations, the pixels are copied into the unknown area from the best matching patch. In order to ensure better propagation of information, the patches are filled in an order of priority. The patches with more known values results in better matching, the patches with more edges results in better structure propagation and the presence of boundary pixels in more levels signifies the persistence at various levels. These factors decide the priority of filling and are termed as confidence term, data term and scale factor respectively. The confidence term and data term imitate the ones used by Crimsini in [24]. Let $\Psi_{\mathrm{p}}$ be the patch of size $n \times n$ centered at point $p$ for some $p \in \delta \Omega$ as shown in Fig.2. The terms for the patch are calculated using Eqn. (4),(5)and (6). The effect of each term is briefly discussed below.

3.1.1Confidence term $(C(p))$ : Patches at the corners and thin protrusions of the target region has more known pixels from the original image. On filling such patches first tend to give more reliable information. Hence boundary pixels are given higher confidence values whereas inner missing pixels are given lesser confidence values. The confidence values for the known area and inpainting area are initialized as 1 and 0 respectively. For a patch centered at ' $p$ ' the confidence term is calculated using Eqn.(4) 


$$
C(p)=\frac{\sum_{q \in \Phi I \Psi_{p}} C(q)}{\left|\Psi_{p}\right|}
$$

3.1.2 Data term $(D(p))$ : Patches that include more edges in the known area will tend to fill first. This factor is a measure of the edge information present in a patch. It helps in preserving linear structures and, ensures the structure propagation into the target region. The values in the known area are initialized to the gradient magnitude. The term for a patch centered at 'p' is then calculated using Eqn. (5)

$$
D(p)=\frac{\sum_{q \in \Phi \mathrm{I}} D(q)}{\left|\Psi_{p}\right|}
$$

3.1.3 Scale factor $(S(p))$ : The unknown pixel that is persistent at various resolution levels tend to fill first. This term measures the existence of pixels at various levels. The value is initialized to 1 for all pixels in the actual sized image. When a pixel exists at other level its value is incremented by 1. This helps in filling the coarser information first. For a boundary pixel 'p' it is calculated using Eqn. (6)

$$
S(p)=\frac{\sum_{i=1 \text { to } L} S\left(p_{i}\right)}{L}
$$

The overall priority of the missing pixels are calculated by Eqn.(7),

$$
P(p)=C(p) . D(p) \cdot S(p), \forall p \in \Omega
$$

The patch with highest priority (say $\alpha$ ) is found and filled with best match region from the source region $\Phi$. The best match is identified with the minimum Sum of square difference between the patches. The search for the best match is limited to the locations as specified earlier. After the filling process the boundary gets altered; the confidence and data terms are updated from the best match. The filling process is repeated in the actual sized image until all unknown values are filled.

\subsection{Hierarchical Filling}

This method uses the exemplar technique in a hierarchical manner. Without the hierarchy this method boils down to the traditional exemplar based method. It fills the unknown pixels in the lowest image resolution first which ensures the coarser information being filled first. When the results are taken to the next higher level it results in certain non-boundary pixels being filled. As a result a single larger mask is now broken to many relatively smaller masks. While filling from lower resolution the finer details get added to the coarser information that is filled earlier.

The mask pixels are traced to the lowest resolution level. The confidence and data terms are calculated as specified in the previous section. The overall priority now involves these two terms as the scale factor is implicitly taken care by the top down approach. The patch with maximum priority is chosen (say $\beta$ ) and similar patch is searched in the known area of the same resolution level. The pixels in the equivalent position of the unknown pixels in $\beta$ are copied from the best match. The confidence term and the data term are updated for the newly filled pixels and the process is repeated until all mask pixels in that resolution level are filled. The results are taken to the next higher resolution (lower level) and the filled using exemplar method. This process repeats until the filling is done in the actual sized image.For color images the algorithm is repeated for each color channel. YCbCr color model is adopted. The RGB image is converted to $\mathrm{YCbCr}$ using the Eqn (8),(9) and(10).

$$
\begin{aligned}
& \mathrm{Y}=16+0.257 . \mathrm{R}+0.504 . \mathrm{G}+0.098 . \mathrm{B} \ldots \ldots \\
& \mathrm{Cb}=128-0.148 . \mathrm{R}-0.291 . \mathrm{G}+0.439 . \mathrm{B} \ldots \ldots \\
& \mathrm{Cr}=128+0.439 . \mathrm{R}-0.368 . \mathrm{G}-0.071 . \mathrm{B} \ldots \ldots .
\end{aligned}
$$

\section{PERFORMANCE ANALYSIS}

Experiments have been done on various images for different mask size and shape. Images with different structures and textures are considered for experimentation. The input images are resized to its nearest $2^{\mathrm{n}}$ size. The image with red colour mask, the inpainted result using Partial Differential Equation (PDE) and the inpainted result using exemplar method is shown in Fig 3(a),(b) and (c) respectively. It is evident from the images that PDE based methods result in blurring while the exemplar method reconstructs in a better way.

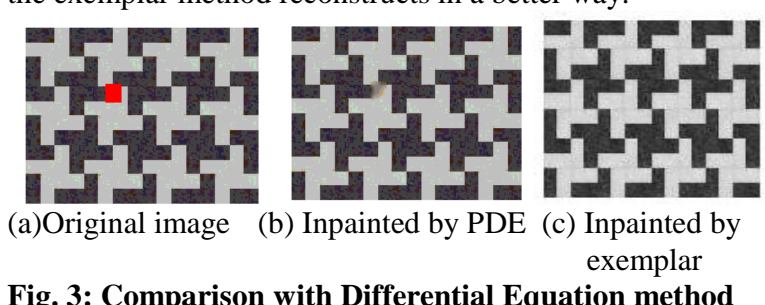

Fig. 3: Comparison with Differential Equation method

Fig 4 has been reproduced from [24], shows the result of exemplar based method on the actual sized image (Level 1). The texture propagated into the area destroying the structure of the roof is indicated in top red circle. The green area propagated into the water body as shown in the bottom red circle.

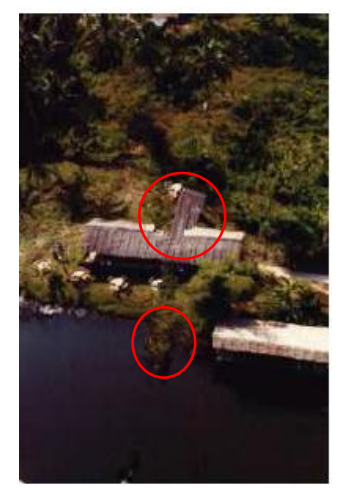

Fig. 4: Inpainted image by exemplar technique

The result of hierarchical method on a similar image is shown in Fig.5. Fig 5(a) and (b) show the original image and the image with mask. Fig5(c) and (d) shows the result of Hierarchical search space refinement and Hierarchical filling respectively. It could be seen from the result that the structure propagation is better in the hierarchical filling than the search space refinement technique. The distortion introduced is slightly higher in the former. 
original image

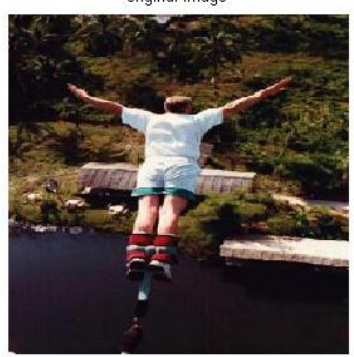

(a): original image

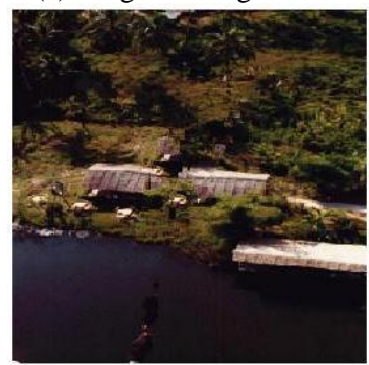

(c): Hierarchical search

space refinement

Fig 5: Inpainted result from Hierarchical methods

The result of Hierarchical filling for an image with linear structure and regular shape is shown in Fig.6 and Fig. 7 respectively. In each case the top row specifies the original image and the bottom row shows the result of Hierarchical filling at various levels. Inpainting from level 1 shows the exemplar based method on the original image. It could be seen that the hierarchical method at level 3 gives better result for image in Fig.6 while reconstruction from level 2 gives a better result for the regular shapes in Fig 7.

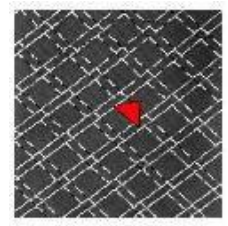

(a): original image

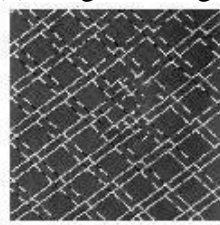

(d):from level 1

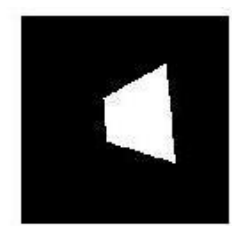

(b): Mask used

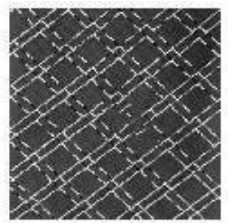

(e): from level 2

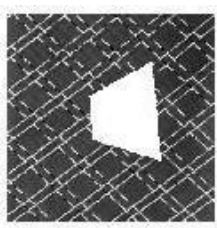

(c)Image with mask

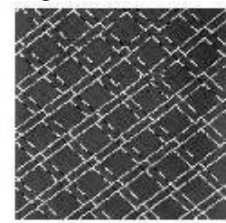

(f):from level3
Fig 6: Inpainted result from Hierarchical Filling at various levels

The performance analysis of inpainting algorithms is usually a subjective process. In order to show the improvement of the algorithm and make the analysis an objective process, an area in the image is chosen by the user. This area is then reconstructed using the inpainting algorithm. Since the image values in the inpainted area is known before reconstruction, the root mean square error can be calculated from the reconstructed pixels and the original pixels. This is used as a metric for the performance analysis.
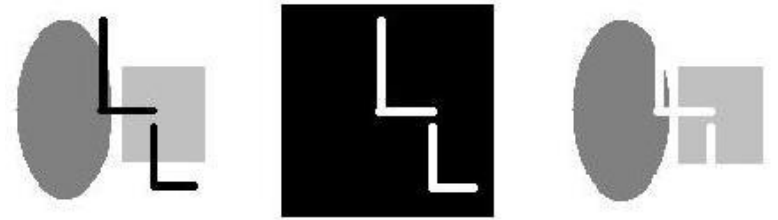

(a): original image

(b): Mask used

(c)Image with mask
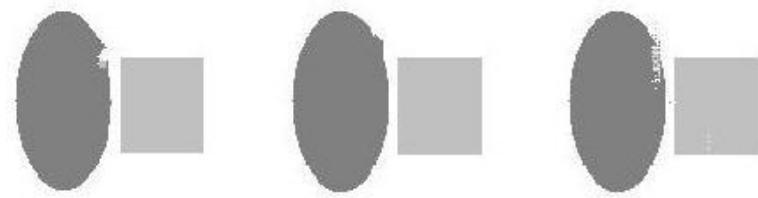

(d): from level 1

(e): from level 2

(f): from level3

Fig 7: Inpainted result from Hierarchical Filling at various levels

In Table 1 the error values are tabulated for the reconstructed image with a) confidence term alone, $\mathrm{C}(\mathrm{P})$, b) with confidence and data term, $\mathrm{C}(\mathrm{P}) \& \mathrm{D}(\mathrm{P})$ and $\mathrm{c})$ with confidence term, data term and the scale factor, $\mathrm{C}(\mathrm{P}), \mathrm{D}(\mathrm{P}) \& \mathrm{~S}(\mathrm{P})$ where the image is reconstructed from 3 different levels.

Table 1: Reconstruction error for various algorithms using Hierarchical search space refinement

\begin{tabular}{|c|c|c|c|c|}
\hline & Levels & $C(p)$ & $\begin{array}{c}C(p) \\
\& D(p)\end{array}$ & $\begin{array}{c}C(p) \& \\
D(p) \& \\
\text { S(p) }\end{array}$ \\
\hline \multirow{3}{*}{$\begin{array}{c}\text { Image } \\
1\end{array}$} & Level 1 & 17.9010 & 16.3014 & 13.8910 \\
\hline & Level 2 & 17.3218 & 15.4017 & 12.1065 \\
\hline & Level 3 & 17.1118 & 14.9018 & 9.9039 \\
\hline \multirow{3}{*}{$\begin{array}{c}\text { Image } \\
2\end{array}$} & Level 1 & 16.9017 & 15.1978 & 12.4190 \\
\hline & Level 2 & 16.0391 & 14.7813 & 10.9034 \\
\hline & Level 3 & 15.3056 & 13.9018 & 9.1945 \\
\hline \multirow{3}{*}{$\begin{array}{c}\text { Image } \\
3\end{array}$} & Level 1 & 15.9201 & 13.5132 & 10.4910 \\
\hline & Level 2 & 15.4804 & 12.8301 & 9.9015 \\
\hline & Level 3 & 15.1190 & 12.0014 & 8.4015 \\
\hline \multirow{3}{*}{$\begin{array}{c}\text { Image } \\
4\end{array}$} & Level 1 & 13.1945 & 12.2018 & 10.3015 \\
\hline & Level 2 & 12.8109 & 11.9015 & 10.0034 \\
\hline & Level 3 & 12.5015 & 11.2178 & 9.5016 \\
\hline \multirow{3}{*}{$\begin{array}{c}\text { Image } \\
5\end{array}$} & Level 1 & 17.1309 & 15.3407 & 12.1078 \\
\hline & Level 2 & 16.3012 & 15.1195 & 11.1841 \\
\hline & Level 3 & 16.0187 & 13.9075 & 10.2819 \\
\hline \multirow{3}{*}{$\begin{array}{c}\text { Image } \\
6 \\
\end{array}$} & Level 1 & 12.4857 & 12.0485 & 11.3839 \\
\hline & Level 2 & 11.9474 & 11.4859 & 10.3849 \\
\hline & Level 3 & 10.4642 & 10.2849 & 9.8338 \\
\hline \multirow{3}{*}{$\begin{array}{c}\text { Image } \\
7 \\
\end{array}$} & Level 1 & 9.4748 & 9.1363 & 8.9383 \\
\hline & Level 2 & 8.5467 & 8.4939 & 8.3393 \\
\hline & Level 3 & 8.5371 & 8.3923 & 8.2484 \\
\hline \multirow{3}{*}{$\begin{array}{c}\text { Image } \\
8\end{array}$} & Level 1 & 10.3747 & 9.9373 & 9.7393 \\
\hline & Level 2 & 9.9383 & 9.7383 & 9.5373 \\
\hline & Level 3 & 9.1939 & 9.0837 & 8.9371 \\
\hline \multirow{3}{*}{$\begin{array}{c}\text { Image } \\
9\end{array}$} & Level 1 & 21.3638 & 20.7372 & 19.6336 \\
\hline & Level 2 & 20.3638 & 19.3838 & 18.3636 \\
\hline & Level 3 & 19.3363 & 18.7363 & 17.3637 \\
\hline \multirow{3}{*}{$\begin{array}{c}\text { Image } \\
10\end{array}$} & Level 1 & 23.6471 & 22.7479 & 21.6467 \\
\hline & Level 2 & 22.6737 & 21.3738 & 20.4747 \\
\hline & Level 3 & 21.3673 & 20.7371 & 20.0383 \\
\hline
\end{tabular}




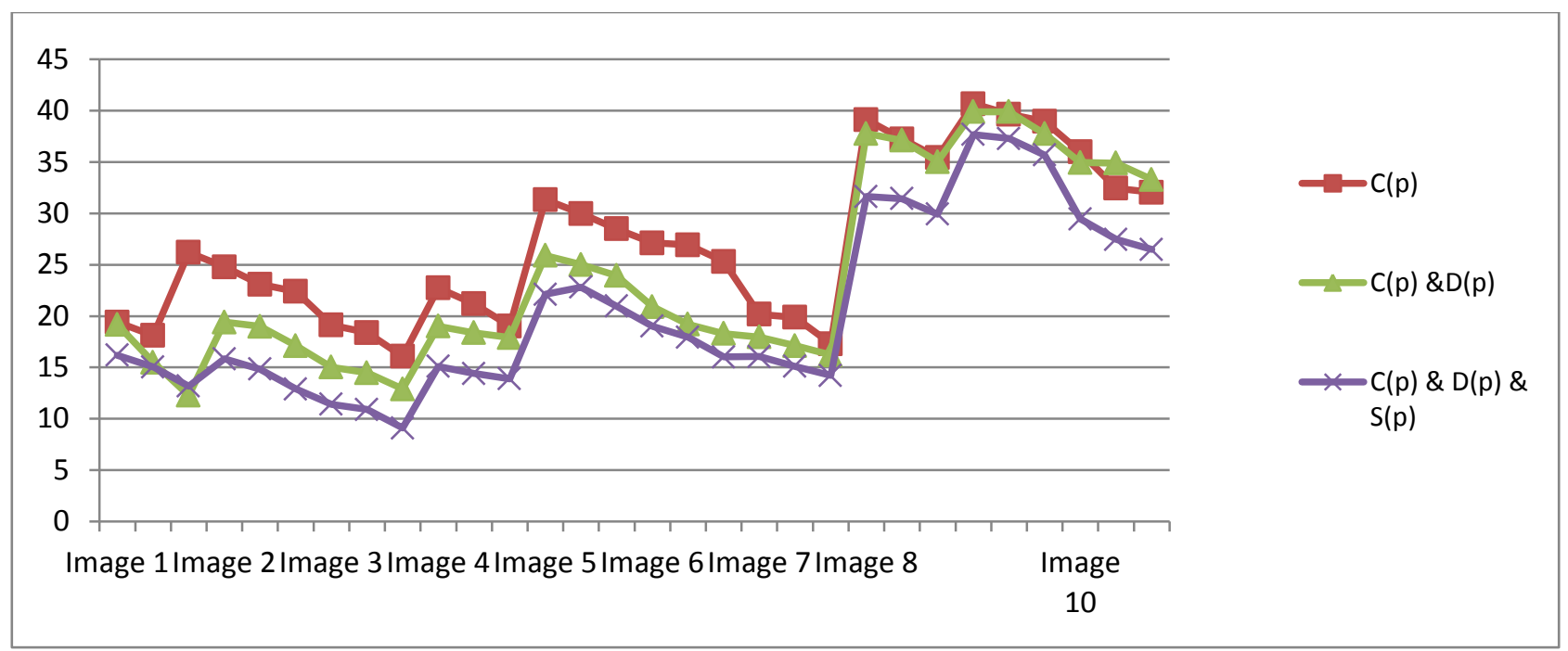

Fig 8: Plot of reconstruction error in Hierarchical search space refinement

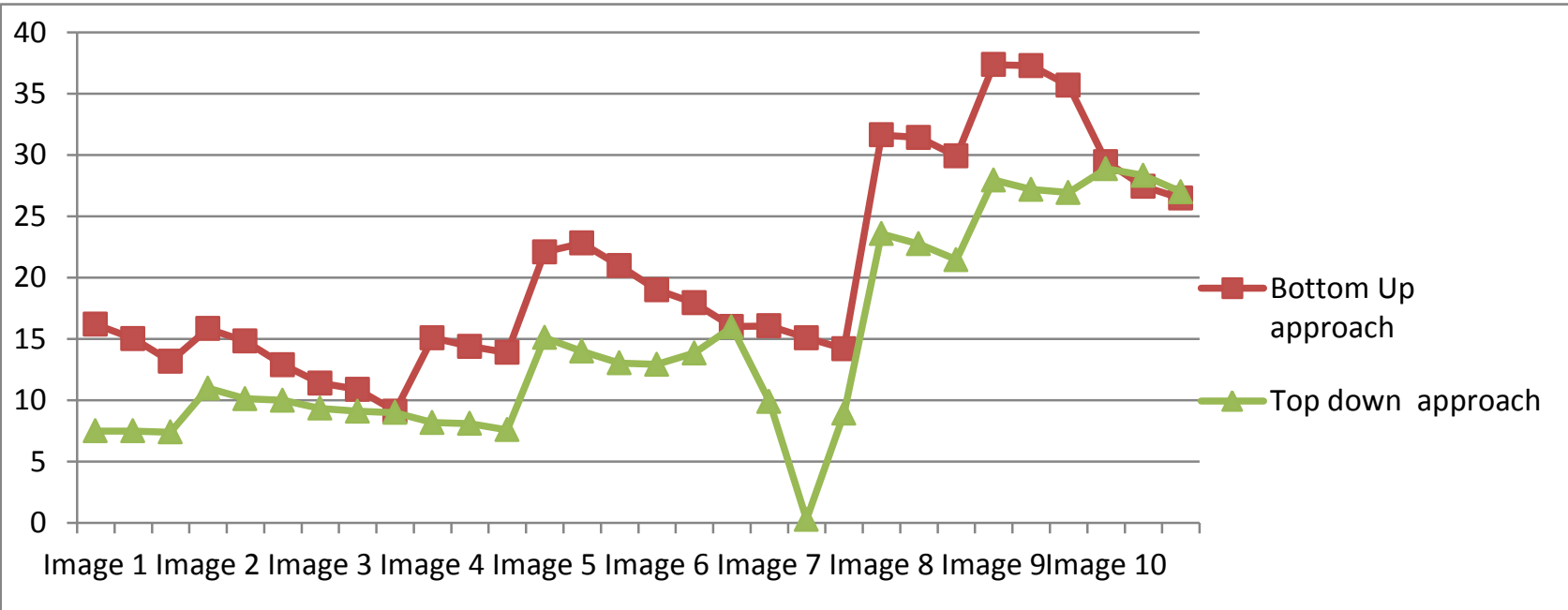

Fig 9: Plot of reconstruction error in Hierarchical search space refinement and Top down Hierarchical filling

The reconstruction is similar to fill front (onion peel) algorithm with confidence term alone. The reconstruction with the confidence and data term specifies the exemplar based method and the third one is the search space refinement algorithm proposed in this paper. Reconstruction at level 1 signifies the algorithms applied on the actual sized images whereas reconstruction from level 2 and 3 specifies the hierarchical method imposed on the corresponding algorithms. A graph plotted for these values is shown in Fig 8 . In the graph, 3 sets of points signifying the level numbers are plotted for each image. From the graph it is clear that the error for Hierarchical search space refinement algorithm is less than the other methods and that the increased level numbers reduces the error further.

The reconstruction error for Hierarchical filling method for 3 different levels is given in Table 2. Filling from level 1 signifies the exemplar based method and the others specify the image reconstructed from the down sampled versions. From the table it can be observed that hierarchical filling reduces the error.
Table 2: Reconstruction error for Hierarchical filling algorithms for various levels

\begin{tabular}{|c|c|c|c|}
\hline & Level1 & Level2 & Level3 \\
\hline Image1 & 19.721 & 18.921 & 18.7826 \\
\hline Image2 & 24.1045 & 24.1023 & 22.4619 \\
\hline Image3 & 26.9017 & 25.1813 & 24.9471 \\
\hline Image4 & 23.0916 & 22.7168 & 21.9036 \\
\hline Image5 & 22.561 & 21.9015 & 18.3719 \\
\hline Image6 & 21.8217 & 21.1059 & 20.539 \\
\hline Image7 & 26.3018 & 25.3018 & 24.8028 \\
\hline Image8 & 26.1637 & 25.9364 & 25.6476 \\
\hline Image9 & 31.9474 & 31.3647 & 30.6478 \\
\hline Image10 & 36.1937 & 35.7464 & 35.5657 \\
\hline
\end{tabular}


Fig.9 shows a plot of reconstruction error for ten different images inpainted from 3 different levels using the Hierarchical search space refinement method mentioned as bottom up approach and the Hierarchical filling method mentioned as top down approach. From the graph it could be seen that Hierarchical filling method performs better in majority of the cases.

\section{CONCLUSION}

Digital image inpainting has a wide application in predicting the unknown portion of the image. Existing methods differ in the propagation of the information into the unknown area and majorly fails when the mask size is large and in natural textured images. This paper addresses this issue using hierarchical approach in two different ways. A search space refinement method looks for a plausible similar match at various resolution while another filling method breaks the larger mask into smaller ones from the lower resolution. The algorithms have been analysed on various images and with varying factors. It is evident from the results that Hierarchical approach improves the quality of reconstruction and that hierarchical filling provides a better solution among the two. The number of levels required for proper reconstruction varies based on the image nature. Since the images are obtained by simple subsampling process distortions occur in the reconstructed image. These distortions are prominent beyond 5 levels for natural textured images and 3 levels for images containing regular shapes. An algorithm that utilizes the nature of the image could be developed for better inpainting.

\section{REFERENCES}

[1] P. PERONA AND J. MALIK, "Scale-Space Edge Detection Using Anisotropic Diffusion", IEEE Transactions on Pattern Analysis and Machine Intelligence, Vol. 12, No.7, July 1990. URL:http://www.cs.berkeley.edu/ malik/papers/MPaniso.pdf

[2] D.J. HEEGER AND J.R. BERGEN "Pyramid-Based Texture Analysis/Synthesis" Proceedings of SIGGRAPH 1995, pp 229-238, September 1995. URL: http://www.cns.nyu.edu/ david/ftp/reprints/heegersiggraph95.pdf

[3] A.C. KOKARAM, R.D. MORRIS, W.J. FITZGERALD AND P.J.W. RAYNER, "Interpolation of Missing Data in Image Sequences",IEEE Transactions on Image Processing. Vol. 4. No.11, Nov. 1995, pp 1509-1519. URL:

http://www.robinmorris.org/sigproc/interpolation.pdf

[4] H. IGEHY AND L. PEREIRA, "Image Replacement through Texture Synthesis", Proceedings of the IEEE International Conference on Image Processing, October 1997.URL:

http://graphics.stanford.edu/papers/texture_replace/textur e_replace.pdf

[5] J.S. DE BONET, "Multi resolution sampling procedure for analysis and synthesis of texture images", in Proc. ACM Conference Computer Graphics (SIGGRAPH), volume 31, pages 361-368, 1997.

[6] V. CASELLES, J. M. MOREL, AND C. SBERT, "An Axiomatic Approach to Image Interpolation", IEEE Transactions on Image Processing, 7, Issue 3, Mar 1998, Page(s): 376 - 386.
[7] M. TUCERYAN AND A. K. JAIN, "Texture Analysis," Handbook of Pattern Recognition and Computer Vision, C. H. Chan, L. F. Pau, and P. S. P. Wang (Eds.), Ch.2, pp. 235-276, Singapore: World Scientific, 1998.

[8] A. EFROS AND T. LEUNG. "Texture synthesis by nonparametric sampling" .In Proc. Int. Conf. Computer Vision, pages 1033-1038, Kerkyra, Greece, September 1999.

[9] M. BERTALMIO, G. SAPIRO, V. CASELLES, AND C. BALLESTER, "Image Inpainting" Proceedings of the ACM SIGGRAPH Conference on Computer Graphics, SIGGRAPH2000, New Orleans, USA. July 2000, pp 417-424.

http://www.iua.upf.es/ mbertalmio/bertalmi.pdf

[10] M. BERTALMIO, A.L. BERTOZZI AND G. SAPIRO, "Navier-Stokes, Fluid Dynamics, and Image and Video Inpainting", Proc. IEEE Computer Vision and Pattern Recognition (CVPR'01), Hawaii, December 2001.http://www.iua.upf.es/ mbertalmio/final-cvpr.pdf

[11] C. BALLESTER, M. BERTALMIO, V. CASELLES, G. SAPIRO, AND J. VERDERA, "Filling-in by Joint Interpolation of Vector Fields and Gray Levels", IEEE Transaction on Image Processing, 10, Issue 8, Aug 2001, Page(s): 1200 - 1211.

[12] M.M. OLIVIEIRA, B. BOWEN, R. MCKENNA AND Y.S. CHUNG, "Fast Digital Image Inpainting", Proceedings of the International Conference on Visualization, Imaging and Image Processing (VIIP 2001), Marbella, Spain 2001. Sep. 3-5, 2001, pp 261266. http://www.cs.sunysb.edu/ oliveira/pubs/inpainting.pdf

[13] T. F. CHAN AND J. SHEN, "Non-Texture Inpainting by Curvature-Driven Diffusions (CDD)", Journal Visual Communication and Image Representation, 12, Number 4, 2001, Page(s): 436 - 449.

[14] M. ASHIKHMIN. "Synthesizing natural textures". In Proc. ACM Symposium on Interactive 3D Graphics, pages 217-226, Research Triangle Park, NC, March 2001.

[15] A. EFROS and W.T. FREEMAN, "Image quilting for texture synthesis and transfer". In Proc. ACM Conf. Comp. Graphics (SIGGRAPH), pages 341-346, Eugene Fiume, August 2001.

[16] A. HERTZMANN, C. JACOBS, N. OLIVER, B. CURLESS, and D. SALESIN, "Image analogies". In Proc. ACM Conf. Comp. Graphics (SIGGRAPH), Eugene Fiume, August 2001.

[17] C. BALleSTER, V. CASELlES, J. VERDERA, M. BERTALMIO, and G. SAPIRO. "A variational model for filling-in gray level and color images". In Proc. Int. Conf. Computer Vision, pages I: 10-16, Vancouver, Canada, June 2001.

[18] P.HARRISON, "A non-hierarchical procedure for resynthesis of complex texture", in Proc. Int. Conf. Central Europe Computer Graphics, Visualization And Computer Vision, Plzen, Czech Republic, February 2001.

[19] S. MASNOU, "Disocclusion: A Variational Approach using Level Lines", IEEE Transactions on Signal Processing, 11, Issue 2, Feb 2002, Page(s): 68- 76. 
[20] R. BORNARD, E. LECAN, L. LABORELLI AND J-H. CHENOT, "Missing Data Correction in Still Images and Image Sequences", ACM Multimedia 2002, Juan-lesPins, France, Dec. 2002.URL: http://brava.ina.fr/papers/INA_Raphael_Bornard/RBorna rd_mm2002_preprint.pdf

[21] T.F. CHAN, J. SHEN AND L. VESE, "Variational PDE Models in Image Processing", UCLA Computational and Applied Mathematics Reports 02-61, Dec. 2002.

[22] J. SHEN, "Inpainting and the Fundamental Problem of Image Processing", SIAM News 36(5), June 2003. http://www.math.ucla.edu/ imagers/htmls/internalreport/ ShenSIAM.pdf

[23] M. BERTAlmio, L. VESE, G. SAPIRO AND S. OSHER, "Simultaneous Structure and Texture Image Inpainting",in Proceedings of the 2003 IEEE Computer Society Conference on Computer Vision and Pattern Recognition (CVPR'03), volume 2, June 2003.URL: http://www.math.ucla.edu/ lvese/PAPERS/01211536.pd $\mathrm{f}$
[24] A. CRIMINISI, P. PÉRES AND K. TOYAMA, “Object Removal by Exemplar-Based Inpainting", Proceedings of the 2003 IEEE Computer Society Conference on Computer Vision and Pattern Recognition (CVPR'03).URL:

http://research.microsoft.com/vision/Cambridge/papers/c riminisi_cvpr03.pdf

[25] I. DRORI, D. COHEN-OR AND H. YESHURUN, "Fragment-Based Image Completion", ACM Transactions on Graphics (TOG), volume 22 issue 3, July 2003. URL: http://portal.acm.org

[26] R.C. GONZALES AND R.E. WOODS, "Digital Image Processing", Second Edition, Prentice Hall, Inc. 2002. ISBN: 0-201-18075-8

[27] M. SONKA, V. HLAVAC AND R. BOYLE, "Image Processing, Analysis and Machine Vision", Second Edition, Brooks/Cole Publishing Company, 1999. ISBN: 0-534-95393-X 